\title{
Generating marker stars for 6D optical tracking
}

\author{
Dominikus Gierlach, Agneta Gustus, and Patrick van der Smagt
}

\begin{abstract}
Visually tracking a large number of objects remains a trade-off between accuracy and amount of data. For applications where high accuracy in both position and orientation of points in space is required, optical tracking systems with passive marker systems are suitable. However, the placement of the marker dots remains problematic, as distinguishability between the marker alignments considerably reduces data pre- or post processing, as objects can be identified by the marker geometry and the identity and pose of marked objects does not have to be reconstructed from prior frames. We propose a method which automatically generates a set of unique marker stars in a selectable quantity. The proposed method generates structures which can be built using standard rapid prototyping techniques and allow the measurement of the unconstrained and independent movement of a large number of objects with an optical tracking system. Resulting structures have proven to work well in our experiments.
\end{abstract}

\section{INTRODUCTION}

Motion tracking is a measurement technique used to obtain information about the position and/or the orientation of objects in specific time frames. Getting this information for multiple objects with sufficient accuracy and frame rate allows the reconstruction and reproduction of movements. The information can be used in animation [3], view control, navigation and instrument tracking (especially in medical applications) and object selection and manipulation [9]. Another important use for biomechanics, sports as well as robotics laboratories is the task of mechanical and biomechanical analysis.

There is a large number of available technical systems that can be used for motion tracking, ranging from mechanical sensing with potentiometers over inertial sensing, acoustic sensing, magnetic sensing and radio wave sensing to optical sensing [9]. To obtain high accuracy as well as high frame rates in different setups, the systems most commonly used in human body motion tracking are magnetic or optical tracking systems [5]. Since the former are heavily disturbed around (moving) metal, only the latter are applicable in situations where cooperation with robotic systems is necessary. Optical systems have a broad range of environments they can be used in. Depending on the used camera system and materials of the marker structures, they can be used in lab environments, surgical environments and even outdoors [8].

The authors are with the Institute of Robotics and Mechatronics, German Aerospace Center / DLR Oberpfaffenhofen, Germany. Email \{dominikus.gierlach, agneta.gustus, smagt\}@dlr.de

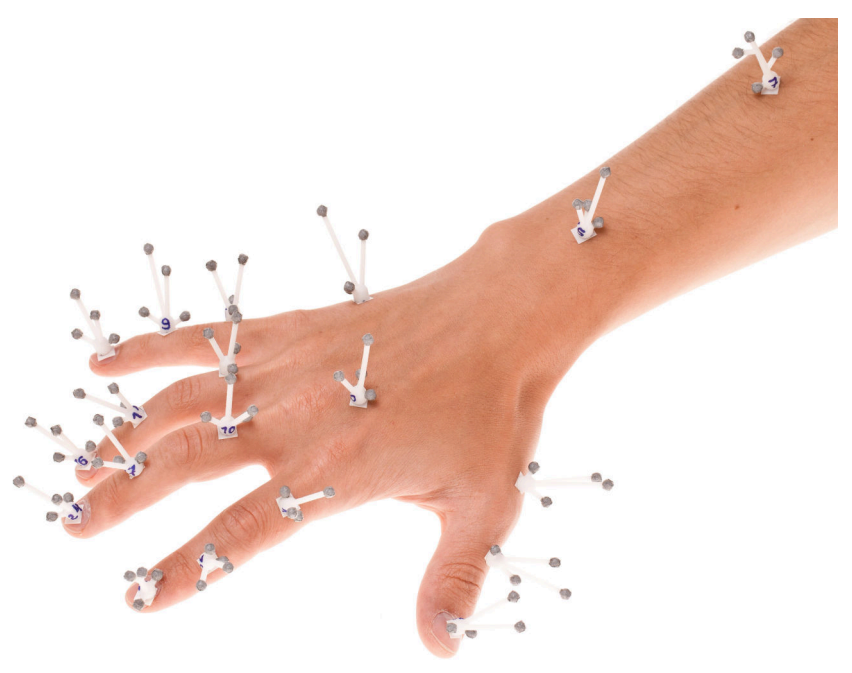

Fig. 1. A hand packed with marker stars

Marker-based optical tracking systems can be divided into two classes: those using active markers and those with passive markers. In the first case, pulsing emission of IR signals from the markers allows for a perfect identification of each marker by the camera systems. There is a major disadvantage, though: Each marker needs to contain electronics and requires an energy supply, leading to a relatively large size and limitation of usability. It is because of this drawback that many systems revert to passive markers. In those setups, the cameras typically emit IR light which is reflected by markers. These are usually spherical to have the same projection from every viewing angle.

By arranging a set of passive markers in a fixed way with no inner symmetries, the tracking system can use such known structures (hence referred to as "marker stars") to not only determine the position but also the orientation of the structure in space.

In this paper we address the problem of identifying and tracking a large number of different markers. Unique labeling of multiple markers with a passive optical tracking system can only be realized by geometrically "sufficiently different" markers. Depending on the tracking system, this difference criterion may vary, but it requires structures with geometries that are unique in three-dimensional arrangement. Finding unique structures "manually" is difficult and non-intuitive. Resulting structures tend to be large and inner symmetries can inadvertently be overlooked.

In this paper we propose a method to generate multiple 
marker stars, given the size of a single marker, a minimum variation between the geometrical arrangements, and the maximum dimensions of the bounding box in which each marker star should fit (i.e. its maximal size). Our method maximizes the differences between pairs of marker stars, in order to optimize the unique recognizability of each one, taking the constraints into account.

We have exploited our proposed method to generate marker stars to detect the position and orientation of single phalanges of the human hand (Fig. 1). In this problem, in order not to disturb free finger movement we chose the bounding boxes to be $20.8 \times 12.6 \times 48.3 \mathrm{~mm}$ in size. We were able to recognise and uniquely label the required 19 marker stars using a six-camera Vicon T10 system with $6 \mathrm{~mm}$ lenses which were placed at $1 \mathrm{~m}$ to $2 \mathrm{~m}$ distance to the hand. A total of 25 unique configurations with partially different mounting options were created, so that the marker stars could be attached to differentsized hands.

We also created sets of larger markers for fixation with Velcro straps for multi-purpose measurements with larger objects. Thus we can employ these marker stars for tracking arms or legs, or indeed any object.

\section{PROBLEM DEFINITION}

Our goal is to track the position and orientation of a large number of different objects. The tracking system recognizes objects which are marked with retroreflecting spheres. Three spheres defined as an object form a triangle, which the system can use to recognize and identify the object, as well as determine its position and orientation. To recognize an object and accurately compute its position and orientation, the triangles are not allowed to have inner symmetries. This means that all three sides defining a triangle have to be of different length. To be able to identify different objects, they are not allowed to have congruent triangles. The triangles of two objects must vary in at least one side.

In order to increase the performance of the system under all conditions with a limited set of cameras, it is preferable to mark objects with four spheres, forming a marker star. Using four spheres provides redundancy, the object can be tracked even if one sphere is not visible to the cameras. If all spheres are visible to the cameras, the tracking accuracy is improved. The spheres are the vertices of an irregular tetrahedron. The bounding areas of the tetrahedron are four scalene, unique triangles, which can be identified and used by the camera system.

The absolute size and shape of a marker star is limited by the requirements for the measurement. In certain environments, e.g. when tracking fingers of the human hand, the size of a marker star has to be very small to prevent disturbances of the measurement. Alternatively, the shape of a star is restricted only in a few of its Cartesian dimensions, e.g. it has to be flat or slim, while the covered area or the height of the star is of lesser relevance.
Finally, depending on the experiment, a certain number of differentiable marker stars is required. To optimize the recognition rate of a large number of stars, we need to aim for maximum differentiability by maximizing asymmetries between stars.

Existing work deals with the problem of optimal tracking accuracy for specific marker geometries of optical and electromagnetic marker stars, but does not handle the problem of tracking a large number of objects with small marker star dimensions [1], [10]. Pintaric and Kaufmann proposed a method to generate rigid body targets for optical tracking systems that focuses on practicability and a simple, manual production method, but does not allow for the creation of distinguishable marker stars that fulfil certain geometric requirements in the quantity we need [4].

\section{METHOD}

Preliminary experiments with a Vicon MX3 camera system showed that the variation of one side of a triangle by a larger value is to be preferred over the variation of several sides of the triangle by a smaller value. We systematically varied the geometry of triangles consisting of $3 \mathrm{~mm}$ markers and examined the ability of the system to distinguish between varied triangles. The camera system uses the center of the tracked markers as position value, so this finding is valid for different marker diameters. We used this finding to develop a method for designing marker stars by maximally varying one edge of a marker star triangle.

\section{A. Definitions}

The size of the working space a marker star is allowed to fill is limited by the requirements of the planned experiment, which we define as bounding box $\left(h_{1}, h_{2}, h_{3}\right)$.

We define a tetrahedron by its non-intersecting edges $(u, U),(v, V)$, and $(w, W)$. The order of the sides in the 3 pairs is important for the definition of a tetrahedron; however, the tetrahedrons defined by $(u, U),(v, V)$, $(w, W)$ is identical to $(U, u),(V, v),(W, w)$ are the same. Fig. 2 depicts our definitions.

We furthermore take into account the following observations:

- Two tetrahedrons are unique when they differ at least in both lengths of one of the pairs of opposed sides. The change of both lengths of one pair affects all the triangles in a tetrahedron, creating a new distinguishable tetrahedron.

- A tetrahedron consists of 4 triangles defined by each length of the pairs $(u, U),(v, V)$, and $(w, W)$. To ensure that all triangles are non-symmetric, each of the pairs $(u, U),(v, V)$, and $(w, W)$ have to differ in their lengths. To ensure that all triangles are unique and the tetrahedron has no isometries, each side of a tetrahedron is different from every other side in the tetrahedron. (Note that we could allow equality in one of the three pairs $(u, U),(v, V)$, or $(w, W)$. 
That would, however, prevent our subsequent observation:)

- It is possible to create a new, unique tetrahedron by changing the order of one of the pairs of sides. For example, if a tetrahedron with the pair $(u, U)$ is changed to a tetrahedron with the pair $(U, u)$, the two resulting tetrahedrons share no isometries.

\section{B. Approach}

The different tetrahedron configurations are built from a pool of sides with different, valid lengths. The sides in the pool differ by a constant value that can be adjusted to the needs of minimal variety of the marker stars and the required number of marker stars in a certain space. Increasing the minimum variation between two sides in order to maximize the determination rate and accuracy of the used tracking system decreases the number of valid, varied tetrahedrons.

We start with a valid, minimum size initial tetrahedron. Then two opposing edges are changed by a sufficient length, enough to enable the tracking system to separate the new tetrahedron from the previous one.

Next, we verify the volume of the new tetrahedron by finding the oriented bounding box (OBB) to check if it still fits within our problem-defined bounding box.

An OBB defines the smallest possible cuboid in which an object fits. Marker stars that do not fit the requirements are sorted out. We repeat the process until a sufficient number of fitting stars is found.

\section{Generating a tetrahedron in Cartesian space}

The first triangle of the tetrahedron is generated in the $x, y$ plane of the 0 coordinate system. We define the initial edge $u$ of the tetrahedron to be along the $x$-axis, starting from the origin. The end points of that edge form the two vertices $A, B$ of the tetrahedron. Then the third vertex $C$ is defined as one of the two intersecting points of two circles around the vertices $A, B$ with the radius of the edges of the triangle $v, w$ (see Fig. 3).

The remaining vertex $D$ lies at an intersection of the sphere $\boldsymbol{S}_{\boldsymbol{A}}$ with radius $V$ around $A$, the sphere $\boldsymbol{S}_{\boldsymbol{B}}$ with

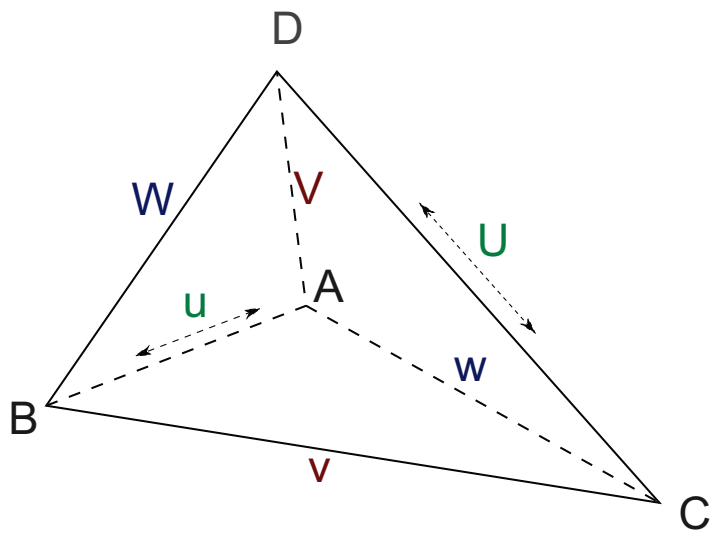

Fig. 2. Tetrahedron, defined by $(\mathrm{u}, \mathrm{U}),(\mathrm{v}, \mathrm{V})$ and $(\mathrm{w}, \mathrm{W})$

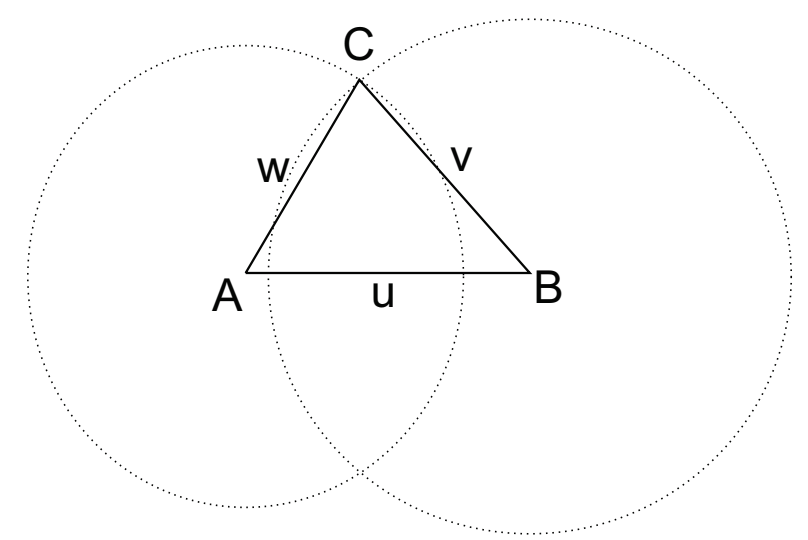

Fig. 3. Generating the tetrahedron from the side lengths. Vertex $C$ is located at the intersection of the two circles with radius $w$ cq. $v$ around $A$ cq. $B$.

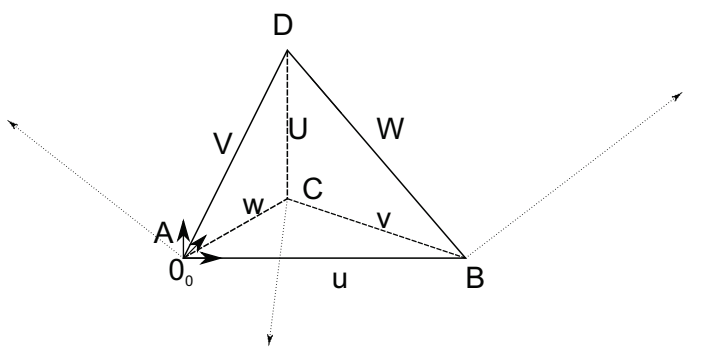

Fig. 4. Generating the tetrahedron from the side lengths. Vertex $D$ is at the intersection of the three spheres with radii $V, W, U$ around $A, B, C$.

radius $W$ around $B$ and the sphere $\boldsymbol{S}_{C}$ with radius $U$ around $C$ (Fig. 4).

In the next step the OBB of the generated tetrahedron is checked.

\section{Finding the Oriented Bounding Box}

There are several methods to find the OBB of an object. We are using a simple method proposed by [2] which uses a 3D covariance matrix of the distribution of vertices of the tetrahedrons to get the orientation of the OBB. The method is not optimal for isometric objects and objects with inner points, but our tetrahedrons neither have inner points nor isometries, so the method suffices for our needs.

We define an oriented bounding box by its center $c$, the halves of the lengths of the boxes' sides $a_{1}, a_{2}, a_{3}$, and its orientation, given by the columns of the rotational matrix ${ }^{0} \boldsymbol{R}_{\mathrm{OBB}}$, formed by $\boldsymbol{n}_{\mathbf{1}}, \boldsymbol{n}_{\mathbf{2}}$, and $\boldsymbol{n}_{\mathbf{3}}$. These vectors are aligned with the face normals of the OBB (see Fig. 5). The matrix ${ }^{0} \boldsymbol{R}_{\mathrm{OBB}}$ describes the rotation from the coordinate system of the OBB to the original coordinate system. 


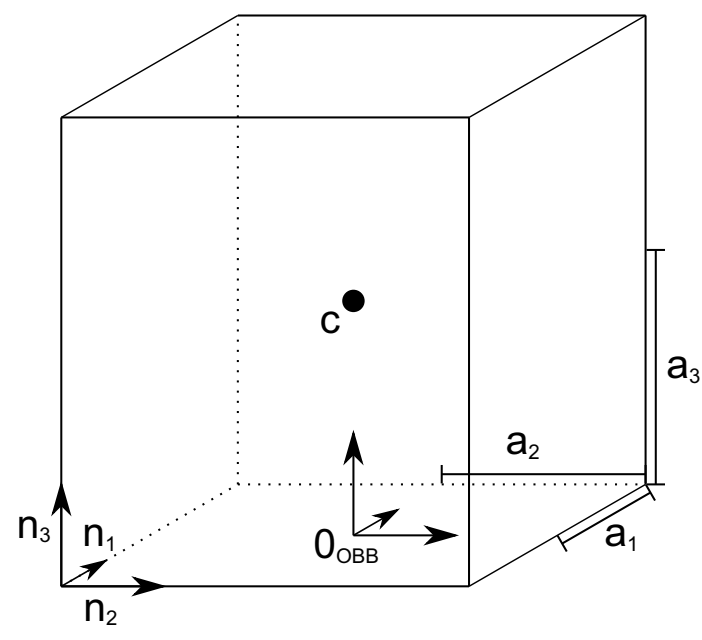

Fig. 5. The Oriented Bounding Box

\section{E. Finding the transformation matrix}

The tetrahedron is generated in the coordinate system 0 in a way that does not place it optimally in our work space. We want the tetrahedron to be placed so that the width of the marker is minimal and the length of the tetrahedron is the second smallest dimension. Minimizing the height of the marker is of secondary interest.

To achieve the optimal placement of the tetrahedron, we have to transform the coordinates. The resulting coordinate system $\mathrm{OBB}$ has the $z$-axis parallel to the longest edge of the $\mathrm{OBB}$, the $y$-axis parallel to the shortest edge of the OBB, and the $x$-axis oriented so that a right-hand system is generated. The origin of this coordinate system lies in the center of the bottom area of the OBB.

The coordinates have to be translated about the vector $-\boldsymbol{c}$, so that the center of the OBB lies in the origin of the 0 coordinate system. Then the coordinates have to be rotated with the rotation matrix ${ }^{\mathrm{OBB}} \boldsymbol{R}_{0}$, so that the orientation of the OBB fits the stated requirements. Then the coordinates have to be translated by half of the length of the longest edge $a_{3}$, so that the smallest bounding area of the OBB lies in the $x y$-plane of the 0 coordinate system.

The orientation of the OBB is to be stored in a rotation matrix ${ }^{0} \boldsymbol{R}_{\mathrm{OBB}}$, which transforms from the OBB coordinate system to the $\mathrm{OBB}$ to the original, 0 coordinate system.

The translation by $-\boldsymbol{c}$ before the rotation and $a_{3}$ in $z$-direction after the rotation can be combined in the translation vector $\boldsymbol{t}$ :

$$
\begin{gathered}
{ }^{0} \boldsymbol{R}_{\mathrm{OBB}}=\left[\begin{array}{lll}
\boldsymbol{n}_{1} & \boldsymbol{n}_{2} & \boldsymbol{n}_{3}
\end{array}\right] \\
{ }^{\mathrm{OBB}} \boldsymbol{R}_{0}={ }^{0} \boldsymbol{R}_{\mathrm{OBB}}^{T} \\
\boldsymbol{t}=-{ }^{\mathrm{OBB}} \boldsymbol{R}_{0}\left(\boldsymbol{c}-a_{3} \boldsymbol{n}_{3}\right)
\end{gathered}
$$

With the translation vector $\boldsymbol{t}$ and the rotation matrix ${ }^{\mathrm{OBB}} \boldsymbol{R}_{0}$ the homogeneous transformation matrix ${ }^{\mathrm{OBB}} \boldsymbol{T}_{0}$ can be formed:

$$
{ }^{\mathrm{OBB}} \boldsymbol{T}_{0}=\left[\begin{array}{cc}
{ }^{\mathrm{OBB}} \boldsymbol{R}_{0} & \boldsymbol{t} \\
(0,0,0) & 1
\end{array}\right]
$$

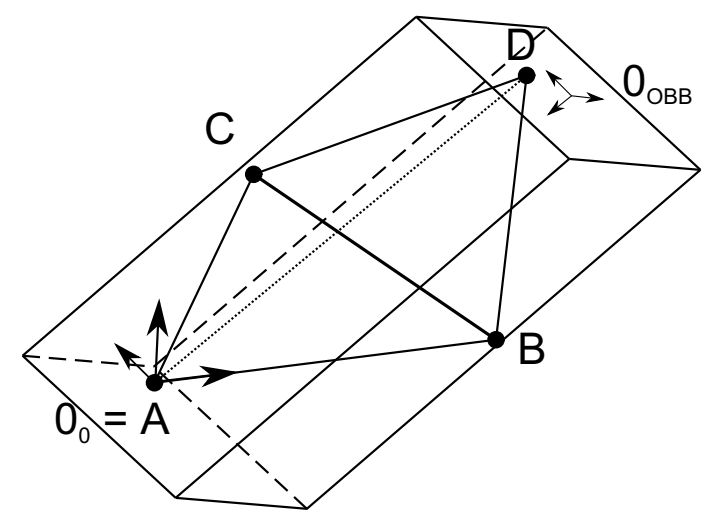

Fig. 6. Generated tetrahedron in the OBB reference

\section{REALIZATION}

The method was implemented using MATLAB. The marker stars were designed using the CAD program Pro/ENGINEER Wildfire v4 and produced with selective laser sintering.

\section{A. Design}

Different marker star configurations were realized by importing a formatted MATLAB table into a Pro/ENGINEER family table with marker coordinates.

Different bases of the marker stars have been realized to provide suitable means of fixing the marker stars to objects. The spheres of the raw models have a diameter of $3.8 \mathrm{~mm}$ and are to be coated in retro-reflective foil so that the approximate diameter of the spheres is $4 \mathrm{~mm}$.

\section{B. Results}

Having determined the size of the bounding box which may not be exceeded by the marker star, our method can be used to compute a desired number of marker stars,

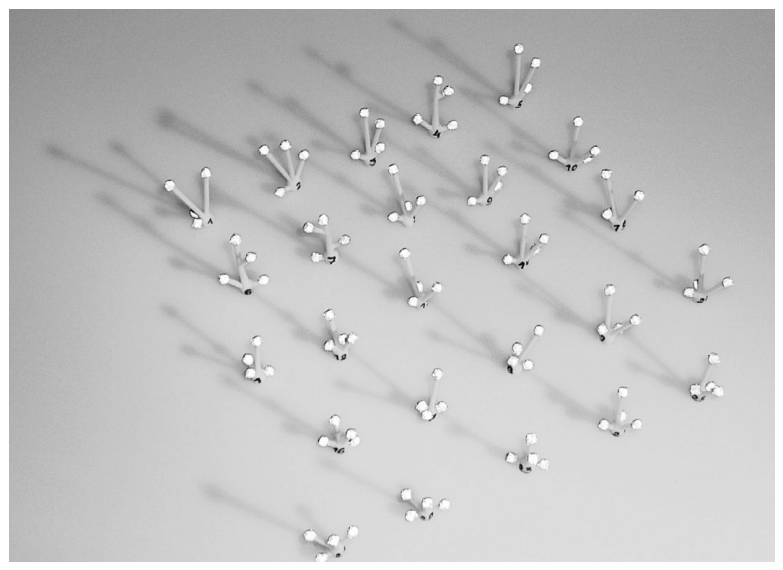

Fig. 7. Complete set of 25 different marker stars 


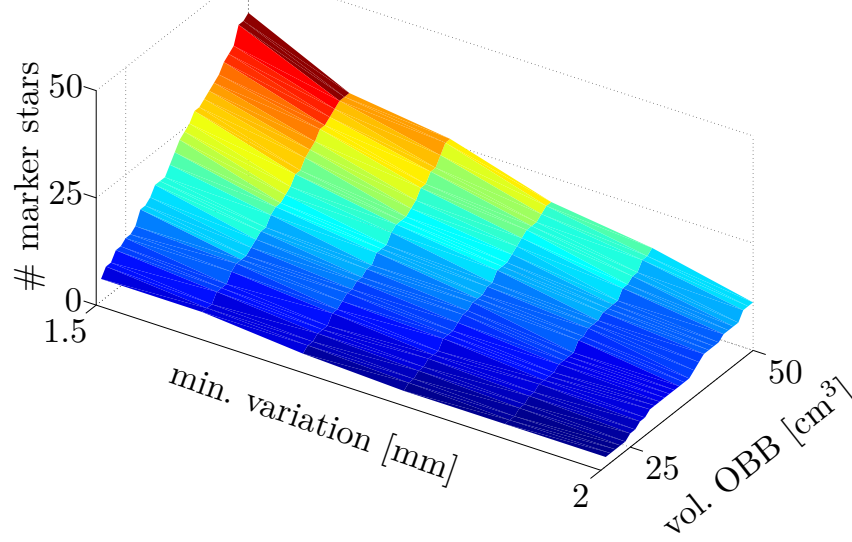

Fig. 8. Number of different marker stars with $4 \mathrm{~mm}$ spheres, depending on the minimal variation of side lengths and the minimal size of a cubic OBB.

depending on the size of a single marker and the minimal variation of at least one side of each triangle in the marker stars, that the optical tracking system requires to distinguish between triangles. The latter number depends on the used camera system and the experimental setup.

Naturally, the larger the diameter of the single markers, the higher the chance to recognize each one, and thus the performance of the system. When the tracking space is limited, e.g. in case of tracking fingers, the maximum dimensions of a single marker star are limited, and the diameter of the markers must be chosen accordingly.

Taking these limitations into account, we can use our algorithm to compute a model for the maximum number of marker stars that can be generated within a certain cubic bounding box, depending on the size of a marker and the variation between triangles.

This is depicted for cubic bounding boxes in Fig. 8 for different minimal variations between triangle side lengths, if markers with $4 \mathrm{~mm}$ diameter are chosen.

Non-cubic bounding boxes change this relationship, as most of the resulting tetrahedrons have a tendency to be prolated because of the differences in their side lenghts. The OBB of a marker star has a smaller volume than the smallest cubic bounding box it fits in. For our problem, in a defined workspace of $20.8 \times 12.6 \times 48.3 \mathrm{~mm}$ and using markers with a diameter of $4 \mathrm{~mm}$ while varying side lengths by at least $1.5 \mathrm{~mm}$, we can create 25 configurations.

The resulting structures have a number of advantages over existing methods to track bones of the hand in biomechanical studies. [7] also tried to measure hand kinematics in $6 \mathrm{DoF}$. Therefore three $3 \mathrm{~mm}$ half spheres were placed on each segment of one finger manually, attempting to arrange the markers in unique geometries. The relative geometry of the markers on the fingers had to be labeled manually. Additionally a reference triangle was placed on the back of the hand in order to be able to combine single finger measurements and obtain the kinematics of the complete hand. It was not possible to track all fingers at once because the triangles were not distinguishable on all segments and the post-processing would have been extremely work intensive. Another effect of placing three markers on skin to obtain a segment pose is deformation of the triangle because of skin movement.

Our proposed method of creating unique and distinguishable structures with small dimensions allows simultaneous tracking of all fingers of a hand. The marker stars are identified and labeled automatically. The stars have a fixed geometry of the marker relative to each other which is not influenced by skin movement. Nevertheless the attachment point of the marker star is still affected by skin movement. The structures can be equipped with flexible means of fixation. This reduces the preparation effort of measurements.

\section{Our Aim}

The marker stars were created in order to allow us to perform accurate 6-D tracking measurements of all phalanxes of a human hand with a Vicon T10 camera system. We use the measured data to create kinematic models of the human hand [6].

The marker stars are used to identify every marked part of the hand and give its position and orientation. We can distinguish up to 25 marked objects, which allows us to mark every single one of the 15 phalanxes of a human hand, add multiple markers to the back of the hand and track the lower arm during the measurements. The tracked lower arm and back of the hand can be used to gain a base system to describe the position and orientation of the hand and its phalanxes.

\section{REFERENCES}

[1] Gregory Scott Fischer. Electromagnetic tracker characterization and optimal tool design (with applications to ENT surgery). Master's thesis, Johns Hopkins University, 2005.

[2] Stefan Gottschalk. Collision Queries using Oriented Bounding Boxes. PhD thesis, Chapel Hill, 2000.

[3] L. Herda, P. Fua, R. Plaenkers, R. Boulic, and D. Thalmann. Skeleton-based motion capture for robust reconstruction of human motion. In Proc. Computer Animation, pages 77-83. IEEE Computer Society, 2000.

[4] Thomas Pintaric and Hannes Kaufmann. A rigid-body target design methodology for optical pose-tracking systems. Proceedings of the 2008 ACM symposium on Virtual reality software and technology, pages 73-76, 2008.

[5] J Richards. The measurement of human motion: A comparison of commercially available systems. Human Movement Science, 18(5):589-602, 1999.

[6] Georg Stillfried and Patrick van der Smagt. Movement model of a human hand based on magnetic resonance imaging (MRI). In International Conference on Applied Bionics and Biomechanics (ICABB), 2010.

[7] Frank van der Hulst, Simon Schätzle, Carsten Preusche, and Andre Schiele. A Functional Anatomy Based Kinematic Human Hand Model with Simple Size Adaptation. In IEEE International Conference on Robotics and Automation (ICRA), 2012.

[8] Vicon Motion Systems. Product info. http://www.vicon.com/products/tseriesoutdoor.html, $18 / 04 / 2012$. 
[9] Greg Welch and Eric Foxlin. Motion tracking: No silver bullet, but a respectable arsenal. IEEE Computer Graphics and Applications, 22:24-38, 2002.
[10] J.B. West and Jr. Maurer, C.R. Designing optically tracked instruments for image-guided surgery. Medical Imaging, IEEE Transactions on, 23(5):533 -545, 2004. 